\title{
Bistability of optically-induced nuclear spin orientation in quantum dots
}

\author{
A. Russell, ${ }^{1}$ Vladimir I. Fal'ko, ${ }^{1}$ A. I. Tartakovskii, ${ }^{2}$ and M. S. Skolnick ${ }^{2}$ \\ ${ }^{1}$ Department of Physics, University of Lancaster, Lancaster, LA1 4YB, UK \\ ${ }^{2}$ Department of Physics and Astronomy, University of Sheffield, S3 7RH, UK
}

(Dated: November 5, 2018)

\begin{abstract}
We demonstrate that bistability of the nuclear spin polarization in optically pumped semiconductor quantum dots is a general phenomenon possible in dots with a wide range of parameters. In experiment, this bistability manifests itself via the hysteresis behavior of the electron Zeeman splitting as a function of either pump power or external magnetic field. In addition, our theory predicts that the nuclear polarization can strongly influence the charge dynamics in the dot leading to bistability in the average dot charge.

PACS numbers: 73.21.La, 72.25Fe
\end{abstract}

The hyperfine interaction in solids between the electron and nuclear spins [1] leads to the Overhauser energy shift $\delta$ of the electron spin states, produced by the magnetic dipole moments of orientated nuclear spins, often described in terms of the effective nuclear magnetic field $B_{N}=2 \delta / g_{e} \mu_{B}$. The hyperfine interaction is also responsible for the transfer of spin from electrons to nuclei and may lead to a significant nuclear spin polarization $S$, if the system is pumped with highly polarized electrons [2, 3, 4]. Recently, nuclear spin effects have been observed in optically pumped semiconductor quantum dots (QDs) $[5,6,7,8,19,10,11,12]$. In these experiments circularly polarized light generated electron-hole pairs which, then, relax into the ground state of the dot, with electrons exhibiting a longer spin memory than the holes (which lose their spin polarization due to a stronger spin-orbit coupling [14, 15]).

Recently, the nuclear spin orientation in optically pumped dots has been found to display a pronounced bistability in externally applied magnetic fields 10, 11, 12]. This appears as a threshold-like switching of the nuclear magnetic field $B_{N} \sim 2-3 T$ and a characteristic hysteresis behavior observed in the dependence of the nuclear polarization on either the intensity of the polarized light 10, 11] or external magnetic field [12, 13].

In this Letter we propose a theory of the nuclear polarization bistability in optically pumped QDs. We study the dynamics of nuclear spins in a dot populated by electrons (el) and holes (h) which arrive into its ground state with the independent rates $w(\mathrm{el})$ and $\tilde{w}(\mathrm{~h})$ and polarization degrees $\sigma$ (el) and $\tilde{\sigma}$ (h) (see Fig. 11(a)). It has been recently noticed that nuclear polarization bistability may occur in the regime when light generates $100 \%$ spin-polarized excitons on the dot. Here, we demonstrate that bistability is a general phenomenon possible in a wide range of experimental conditions, including the nonresonant excitation conditions and in the regime when a dot often appears in a positively charged (trion) state. We also predict a new phenomenon caused by the bistable behavior of the nuclear spin orientation: the bistability of the dot average charge.

In optically pumped dots, nuclear spins become orientated due to the spin flip-flops in which a single electron
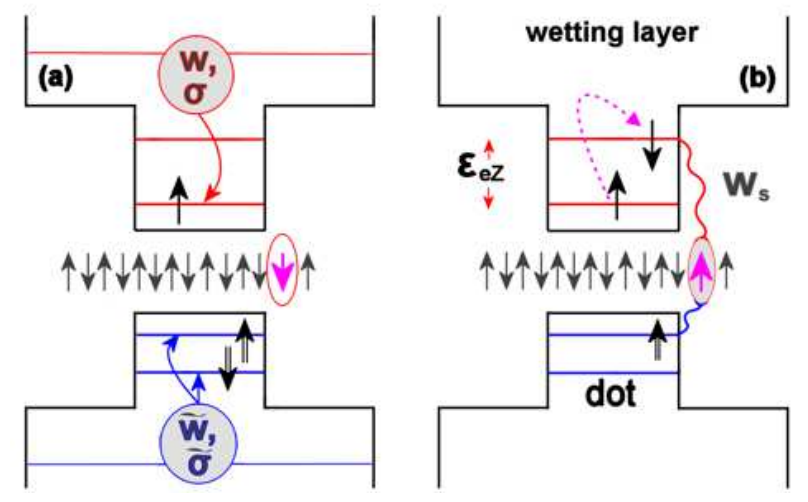

FIG. 1: (a)Diagram of optical excitation of the dot. The case of strongly polarized electrons and weakly polarized holes is shown. (b) Spin-flip-assisted el-h recombination accompanied by flipping one of the nuclear spins.

and one of the nuclei exchange spins via the hyperfine interaction. The process leading to spin transfer consists of an el-nucleus spin flip-flop accompanying the recombination of a polarized electron with a spin $\pm \frac{3}{2}$ heavy hole in a QD carrying a neutral or positively charged exciton (see Fig. 1(b)). In such a process, the electron occupies the intermediate inverted-spin state on the dot virtually, since in a magnetic field a real single-electron spin-flip is prohibited by energy conservation and the Zeeman splitting. The rate of the electron spin-flip recombination involving a single nucleus [17] is,

$$
w_{s}=|u|^{2} w_{r} /\left(\epsilon_{e Z}^{2}+\frac{1}{4} \gamma^{2}\right) .
$$

Here $u$ is a typical energy of the hyperfine interaction with a single nucleus, $\gamma$ is broadening of the electron energy level, and $w_{r}$ is the rate at which the bright exciton recombines on the dot. The electron Zeeman splitting, modified by the Overhauser field $B_{N}$, is $\epsilon_{e Z}=$ $g_{e} \mu_{B}\left(B-B_{N}\right)$. The form of the Eq. (1) implies a feedback due to the dependence of $w_{s}$ on $B_{N}$, which is key to the nuclear spin bistability.

The kinetic model describing the carrier population in the ground state of the dot is formulated in terms of the probabilities of its 16 allowed configurations based upon 
the two electron and two hole spin states corresponding to the lowest $\mathrm{el} / \mathrm{h}$ orbitals in the $\mathrm{QD}$. We solve the rate equations for the populations of these states and for the nuclear orientation, and then find the steady-state magnitude of the nuclear spin polarization $S$. Here, we denote the probability that the dot is empty by $n$, and use $n_{\mu}\left(n^{\mu}\right)$ for the probabilities of the dot occupation by a single electron (hole), with the index $\mu=+/-$ representing the spin state of the particle. We refer to these states as $D, D_{\mu}$ and $D^{\mu}$, respectively. The probabilities for the dot to be occupied with two electrons or two holes (states $D_{+-}$and $D^{+-}$) are $n_{+-}$and $n^{+-}$. The probability to find the dot in a dark exciton state $X_{\mu}^{\mu}$ is $n_{\mu}^{\mu}$, and in a bright exciton state $X_{\mu}^{-\mu}$ is $n_{\mu}^{-\mu}$. The probability to find the dot in a negative (positive) trion state labelled as $X_{+-}^{\mu}\left(X_{\mu}^{+-}\right)$is $n_{+-}^{\mu}\left(n_{\mu}^{+-}\right)$and, finally, $n_{+-}^{+-}$represents the dot in the biexciton state, $X_{+-}^{+-}$.

Below we list the balance equations for the dot population. The first two equations describe the probability of the dot occupation by a single carrier.

$$
\begin{aligned}
\dot{n}_{\mu}= & \frac{1}{2}(1+\mu \sigma) w n+w_{r} n_{+-}^{\mu}-\left[\tilde{w}+\frac{1}{2}(1-\mu \sigma) w\right] n_{\mu} \\
\dot{n}^{\mu}= & \frac{1}{2}(1+\mu \tilde{\sigma}) \tilde{w} n+w_{r} n_{\mu}^{+-}+\frac{1}{2}(1+\mu S) N w_{s} n_{-\mu}^{+-} \\
& -\left[w+\frac{1}{2}(1-\mu \tilde{\sigma}) \tilde{w}\right] n^{\mu} .
\end{aligned}
$$

Both include "gains" due to the arrivals of an electron/hole into the empty dot (Fig. 1(a)) and the recombination of a charged bright exciton, and "losses" due to the arrival of an electron or a hole. The second equation also has a gain due to a possible spin-flip-assisted recombination from a positive trion $X_{\mu}^{+-} \rightarrow D^{-\mu}$ in which the spin is transferred to a nucleus [16], Fig. 1(b). This process is impossible for a negative trion since in the lowest orbital state the flip-flop is blocked by the presence of the second electron [16]. The probability for an el-h pair to recombine via spin-flip depends on the number of nuclei available, which leads to the term $(1+\mu S) N w_{s} n_{-\mu}^{+-}$in Eq. (2), where $S$ is the degree of nuclear polarization and $N$ is the total number of nuclei covered by the electron wave function $\left(N \sim 10^{4} \div 10^{5}\right.$ in a typical InGaAs/GaAs dot).

Equations describing the QD states $D_{+-}$and $D^{+-}$are:

$$
\begin{aligned}
& \dot{n}_{+-}=\frac{1}{2} \sum_{\mu}(1-\mu \sigma) w n_{\mu}-\tilde{w} n_{+-} ; \\
& \dot{n}^{+-}=\frac{1}{2} \sum_{\mu}(1-\mu \tilde{\sigma}) \tilde{w} n^{\mu}-w n^{+-} .
\end{aligned}
$$

Kinetics of the the neutral bright and dark excitons $X_{\mu}^{\mu}$ and $X_{\mu}^{-\mu}$ are described by

$$
\begin{aligned}
\dot{n}_{\mu}^{\mu}= & \frac{1}{2}(1+\mu \tilde{\sigma}) \tilde{w} n_{\mu}+\frac{1}{2}(1+\mu \sigma) w n^{\mu} \\
& -\frac{1}{2}\left[(1-\mu S) N w_{s}+(1-\mu \sigma) w+(1-\mu \tilde{\sigma}) \tilde{w}\right] n_{\mu}^{\mu} ; \\
\dot{n}_{\mu}^{-\mu}= & \frac{1}{2}(1-\mu \tilde{\sigma}) \tilde{w} n_{\mu}+\frac{1}{2}(1+\mu \sigma) w n^{-\mu}+w_{r} n_{+-}^{+-} \\
& -\left[w_{r}+\frac{1}{2}(1-\mu \sigma) w+\frac{1}{2}(1+\mu \tilde{\sigma}) \tilde{w}\right] n_{\mu}^{-\mu} .
\end{aligned}
$$

Both neutral bright and dark exciton populations decrease when more carriers arrive onto the dot. The neutral bright exciton can also be created and removed due to the el-h pair recombination in the processes $X_{+-}^{+-} \rightarrow$ $X_{\mu}^{-\mu}$ and $X_{\mu}^{-\mu} \rightarrow D$, respectively. The dark exciton can decay due to the spin-flip-assisted recombination $\left(X_{\mu}^{\mu} \rightarrow D\right)$ leading to spin transfer to nuclei [16].

Kinetics of the trions $X_{+-}^{\mu}$ and $X_{\mu}^{+-}$are described by

$$
\begin{aligned}
\dot{n}_{+-}^{\mu}= & \frac{1}{2}(1+\mu \tilde{\sigma}) \tilde{w} n_{+-}+\frac{1}{2} \sum_{\nu= \pm}(1-\nu \sigma) w n_{\nu}^{\mu} \\
& -\left[w_{r}+\frac{1}{2}(1-\mu \tilde{\sigma}) \tilde{w}\right] n_{+-}^{\mu} ; \\
\dot{n}_{\mu}^{+-}= & \frac{1}{2}(1+\mu \sigma) w n^{+-}+\frac{1}{2} \sum_{\nu= \pm}(1-\nu \sigma) w n_{\mu}^{\nu} \\
& -\left[w_{r}+\frac{1}{2}(1-\mu S) N w_{s}+\frac{1}{2}(1-\mu \sigma) w\right] n_{\mu}^{+-} .
\end{aligned}
$$

Both trion populations change due to the recombinations $X_{\mu}^{+-} \rightarrow D^{\mu}, X_{+-}^{\mu} \rightarrow D_{\mu}$ and arrival of a single additional charge (the ground states of the dot permit maximum four carriers). A positive trion can also recombine in the spin-flip-assisted process $X_{\mu}^{+-} \rightarrow X^{-\mu}$, forbidden for the negative trions [16].

Finally, the biexciton state $X_{+-}^{+-}$cannot contribute to the nuclear spin pumping as it decays without the spinflip, $X_{+-}^{+-} \rightarrow X_{+}^{-}, X_{-}^{+}$,

$$
\begin{aligned}
\dot{n}_{+-}^{+-}= & \frac{1}{2} \sum_{\mu}\left[(1-\mu \tilde{\sigma}) \tilde{w} n_{+-}^{\mu}+(1-\mu \sigma) w n_{\mu}^{+-}\right] \\
& -2 w_{r} n_{+-}^{+-} .
\end{aligned}
$$

The probabilities for the dot with a given nuclear polarization to be in each of the 16 configurations are found using the normalization condition $1=n+n_{+-}+n^{+-}+$ $n_{+-}^{+-}+\sum_{\mu} n_{\mu}+n^{\mu}+n_{\mu}^{\mu}+n_{\mu}^{-\mu}+n_{+-}^{\mu}+n_{\mu}^{+-}$and the steady state condition for Eqs. (2 26). We formally write these equations in the form $\hat{M} \vec{n}=(1,0, \ldots, 0)^{T}$, where the components of $\vec{n}$ are the occupation numbers and $\hat{M}$ is a $16 \times 16$ matrix with elements determined by the coefficients in Eqs. (2-6) and the normalization condition. The formal solutions for components of $\vec{n}$ are given by $C_{i, 1} / \operatorname{det} M$ where $C_{i, 1}$ is the relevant cofactor of $\hat{M}$.

A steady-state value for the nuclear polarization $\mathrm{S}$ (defined as $S=f_{\Uparrow}-f_{\Downarrow}$ ) can be obtained by substituting formal steady-state solutions of Eqs. (2) 60) for a given $S$ into the balance equation for the occupation numbers of spin up $\left(f_{\Uparrow}\right)$ and down $\left(f_{\Downarrow}\right)$ nuclei [18],

$$
\dot{S}=I \equiv \sum_{\mu} \mu(1-\mu S)\left(n_{\mu}^{\mu}+n_{\mu}^{+-}\right) w_{s}-2 S w_{d} .
$$

It summarizes the processes leading to the nuclear spin pumping: $S$ is increased as a result of the spin-flipassisted recombination of $X_{+}^{+}$and $X_{+}^{+-}$and reduced due to a similar recombination process involving $X_{-}^{-}$and $X_{-}^{+-}$. Thus the balance between the populations of $X_{+}^{+}$ and $X_{+}^{+-}$on one hand and $X_{-}^{-}$and $X_{-}^{+-}$on the other 


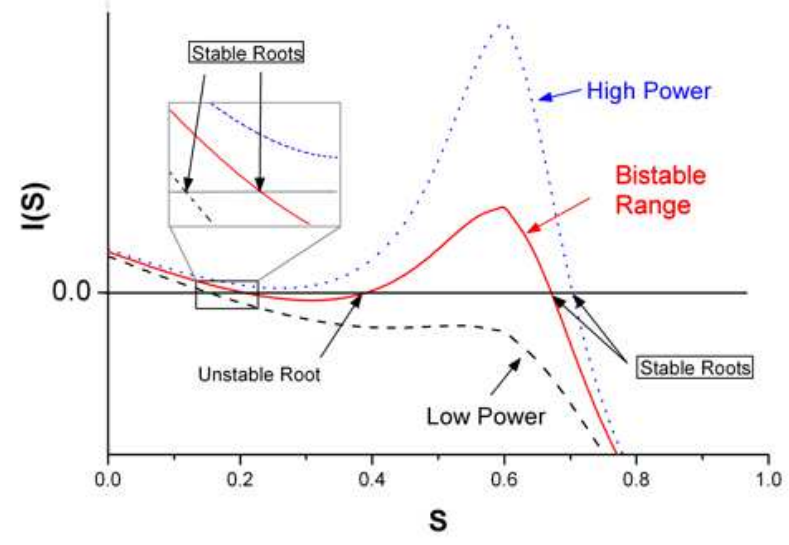

FIG. 2: The function $I(S)$ for the situation where $w=\tilde{w}, \sigma=$ $0.9, \tilde{\sigma}=-0.2, x=0.6$ and $z=8$ for three different powers: $P=0.0001,0.0003,0.0005$. Stable roots correspond to the solutions of $I(S)=0$ where $\frac{d I}{d S}<0$.

will eventually define the sign of the net nuclear polarization [16]. However, an additional important contribution to the depolarization of the nuclei has to be taken into account. It arises from their mutual dipole-dipole interaction effectively leading to the nuclear spin diffusion from the dot into the bulk semiconductor [19], described in our model by the rate $w_{d}[20]$.

To present the analysis of the above equations, we employ the following parameters:

$$
x=\frac{B}{B_{N}^{\max }}, \quad z=2 N^{2} \frac{w_{d}}{w_{r}}, \quad P=\frac{\tilde{w}}{z w_{r}} .
$$

Here $B_{N}^{\max }$ is defined through the Overhauser field $B_{N}$ as $B_{N}=B_{N}^{\max } S$ and, after introducing $\alpha=\gamma / g_{e} \mu_{B} B_{N}^{\max }$, Eq. (11) can be represented in the form [21]:

$$
w_{s} \equiv \frac{w_{r}}{N^{2}(x-S)^{2}+\frac{1}{4} \alpha^{2}} .
$$

The steady-state values of $S$ determined by the feedback built into Eqs. (1.9) are given by the solutions of the equation $I(S)=0$, satisfying the condition $\frac{d I}{d S}<0$ (solutions with $\frac{d I}{d S}>0$ are unstable). Figure2 demonstrates that for a fixed external magnetic field the number of stable solutions for the nuclear spin polarization varies: it can be one or two depending on the incident power and other experimental parameters such as $w_{d}, \sigma, \tilde{\sigma}$ and the ratio $w / \tilde{w}$. At small powers only a single low value of $S$ is possible. At high powers when two stable solutions appear, including one with a large $S>x$, the dot enters the regime of the nuclear spin bistability. This result strongly depends on the depolarization parameter $z$, defined in Eq. (8), so that in the following discussion we specify the range of $z$ where a bistability occurs.

The bottom parts of Figs. 3(a) and (b) show the calculated evolution of the nuclear polarization in a dot for realistic magnitudes of the depolarization parameter $z$ in the regime where electrons have a high degree
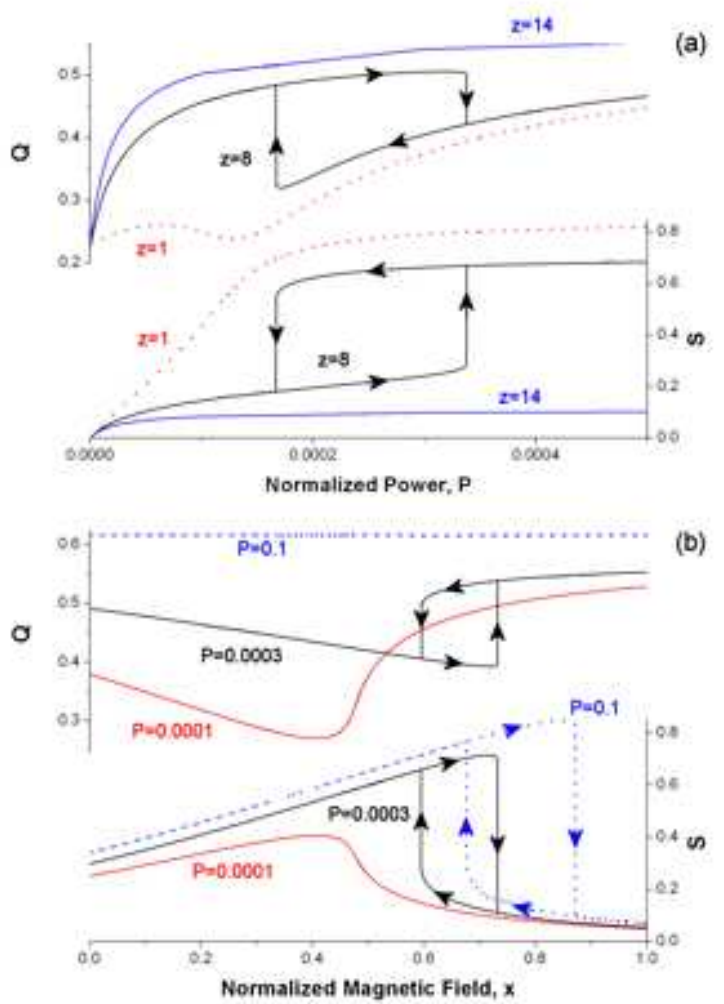

FIG. 3: Evolution of nuclear polarization $(S)$ and the average charging state of the $\operatorname{dot}(Q)$ for $w=\tilde{w}, \sigma=0.9$ and $\tilde{\sigma}=-0.2$ : (a) as a function of power for $x=0.6$ and various values of $z$, with the arrows indicating a forwards or backwards sweep. Although not shown in the figure, at high powers $P \approx 1$, both $Q$ and $S$ start to decrease due to the dot being dominated by the biexciton (for which the spin-flip process is blocked); (b) as a function of magnetic field for $z=8$ and various power values.

of spin memory and arrive with the same rate as the depolarized holes. Fig. 3(a) contains a large hysteresis loop in the power dependence of $S$ for a fixed magnetic field (here, $x=0.6$ ), similar to those observed in Refs. 10,11. The bistable behavior occurs for a wide range of the depolarization parameter $z: 5 \lesssim z \lesssim 14$. Experimentally, the evolution of $S$ can be detected in polarizationresolved PL experiments on individual self-assembled InGaAS/GaAs quantum dots, by deducing it from the measured exciton Zeeman splitting.

We also find that the bistability in $S$ leads to a novel phenomenon: a hysteresis in the average dot charge, $Q$ (see top parts of Fig. 3(a,b)). This occurs when the electrons arriving to the dot have a high degree of spin polarization, permitting their recombination with only one spin orientation of holes. Thus, an extra hole with the opposite spin is likely to remain on the dot, leading to, on average, a positive dot charge. The enhancement of the spin-flip-assisted recombination for large $S$, removing such holes, will result in reduction of the charge. Therefore the hysteresis in $S$ will be reflected as a hysteresis 


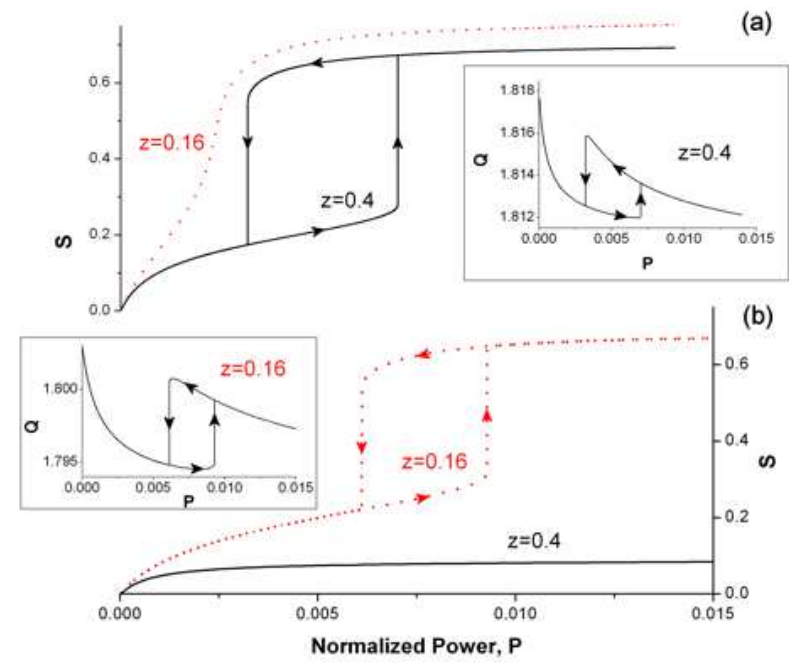

FIG. 4: Evolution of $S$ with $P$ in the regime where $w=$ $0.1 \tilde{w}$ for $z=0.16,0.4$ and different polarizations of arriving electrons/holes. (a) $\sigma=0.9, \tilde{\sigma}=-0.2$. The inset shows the evolution of the charging state of the dot for $z=0.4$ (with a very small hysteresis loop). (b) Same for $\sigma=0.45, \tilde{\sigma}=-0.1$.

in the average dot charge. A similar bistable behaviour in both $S$ and $Q$ can also be found if the external magnetic field is varied [12] at a fixed optical pump power, as shown in Fig. 3(b).

Figure 4 illustrates that the range of parameters for which the bistability can occur strongly depends on the ratio between the arrival rates of electrons and holes, $w$ and $\tilde{w}$, as well as on their polarizations, $\sigma$ and $\tilde{\sigma}$. In experiment the ratio $w / \tilde{w}$ can be varied by applying an electric field in a diode containing QDs in the intrinsic region [4, 6, 11, 12]: because of a light effective mass, electrons can tunnel out before relaxing to the dot ground state, which in effect reduces their arrival rate as compared to that of the holes. Fig. 4(a) shows the evolution of $S(P)$ for $w=0.1 \tilde{w}$. The dot is mainly in the state $D^{+-}$so that its average charge is $Q \approx+1.8$ and exhibits a weak power-dependence with a negligible hysteresis loop (see inset), despite a pronounced hysteresis loop in the nuclear polarization. As seen from the figure, for such low values of $w / \tilde{w}$ higher powers are required to pump a significant nuclear polarization, and the bistability in $S$ is moved towards smaller values of the depolarization parameter $(0.2 \lesssim z \lesssim 0.5)$. Figure 4 (b) illustrates that when the polarizations of both electrons and holes is reduced by $50 \%$ the bistability can still be observed, but only for $0.1 \lesssim z \lesssim 0.2$.

To summarize, we have shown that for a wide range of dot parameters (including the number of nuclei, el-h radiative recombination time and nuclear spin diffusion rate) the polarization of nuclei in a non-resonantly optically pumped semiconductor quantum dot can exhibit a bistable behavior. Thus, we conclude that the nuclear spin bistability is a general phenomenon for dots pumped with circularly polarized light. In addition, we find that the nuclear spin polarization can also strongly influence the charge dynamics in the dot leading to the bistability of the average dot charge.

We thank A. Imamoglu, O. Tsyplyatyev and A. Yacobi for discussions. This work has been supported by the Lancaster EPSRC Portfolio Partnership No. EP/C511743, the Sheffield EPSRC Programme Grant No. GR/S76076, the EPSRC IRC for Quantum Information Processing, ESF-EPSRC network Spico EP/D062918, EPSRC Advanced Research Fellowship $\mathrm{EP} / \mathrm{C} 54563 \mathrm{X} / 1$.
[1] A. W. Overhauser, Phys. Rev. 92, 411 (1953).

[2] D. Pines, J. Bardeen, and C. P. Slichter, Phys. Rev. 106, 489 (1957)

[3] D. Paget et al., Phys. Rev. B 15, 5780 (1977)

[4] P.-F. Braun et al., Phys. Rev. Lett. 94, 116601 (2005).

[5] D. Gammon et al., Phys. Rev. Lett. 86, 5176 (2001); A. S. Bracker et al., ibid. 94, 047402 (2005).

[6] C. W. Lai et al., Phys. Rev. Lett. 96, 167403 (2006).

[7] T. Yokoi et al., Phys. Rev. B 71, 041307 (2005).

[8] I. A. Akimov, D. H. Feng and F. Henneberger, Phys. Rev. Lett. 97, 056602 (2006).

[9] B. Eble et al., Phys. Rev. B 74, 081306 (2006).

[10] P.-F. Braun et al., Phys. Rev. B 74, 245306 (2006).

[11] A. I. Tartakovskii et al., Phys. Rev. Lett. 98, 026806 (2007).

[12] P. Maletinsky et al., Phys. Rev. B 75, 035409 (2007).

[13] Hints of bistability in nuclear orientation have been seen in the spin-polarized tunneling experiments: K. Ono and S. Tarucha, Phys. Rev. Lett. 92, 256803 (2004); S. Tarucha et al., Phsy. Stat. Sol. B 243, 3673 (2006).

[14] T. Uenoyama and L. J. Sham, Phys. Rev. Lett. 64, 25
(1989); T. C. Damen et al., ibid. 67, 24 (1991).

[15] A. Ebbens et al., Phys. Rev. B 72, 073307 (2005).

[16] In the opposite polarization of light, the Overhauser field enhances the Zeeman splitting so that it cannot cause a bistability. Also, virtual spin-flip processes via excited orbital states have a much smaller probability, and are thus neglected. Here we also neglect the effect of the electronhole exchange on the electron spin splitting.

[17] S. I. Erlingsson, Y. V. Nazarov, and V. I. Fal'ko, Phys. Rev. B 64, 195306 (2001).

[18] For simplicity, we consider spin $\frac{1}{2}$ nuclei. Higher spins will result only in the re-parameterization of $N$.

[19] D. Paget, Phys. Rev. B 25, 4444 (1982).

[20] For a dot with radius $r \approx 5 n m$, we approximate $w_{\text {dep }} \approx$ $D_{N} / r^{2} \approx 1-10 s^{-1}$, where $D_{N} \approx \mu_{n}^{2} / \hbar a$ is the coefficient of polarization diffusion due to the dipole-dipole interaction between magnetic moments $\mu_{n}$ of neighbouring nuclei and $a=0.56 \mathrm{~nm}$ is the lattice constant.

[21] For InGaAs quantum dots used in [11], $w_{r} \approx$ $10^{9} s^{-1}, N \approx 10^{4}, B_{N}^{\max } \approx 2-3 T, \alpha \approx 0.01$. 